\title{
RECENSIONES BIBLIOGRAFICAS
}

Trilla, JAime (1985): La educación fuera de la escuela. Enseñanza a distancia, por correspondencia, por ordenador, radio, vídeo y otros medios no formales. Planeta, Barcelona.

La aparición de una serie de factores de índole diversa ha incidido, notoriamente, desde hace ya tiempo, en la estructura social provocando la aparición de nuevas necesidades educativas, produciendo desajustes ostensibles en los sistemas educativos formales y propiciando, a su vez, el auge de los medios no formales de educación. Entre 'estos factores se destacan en el libro los siguientes:

- El incremento de la demanda educativa en una gran diversidad de áreas, muchas de ellas fuera de la enseñanza reglada (formación de adultos, ocio, reciclaje profesional, etc.).

- La crisis económica que ha convertido las soluciones a los problemas educativos desde el sector formal, en una carga excesivamente onerosa. De ahí que los medios no formales representen una alternativa más rentable desde un punto de vista económico, aunque sea un criterio insuficiente para decidir el orden de prioridades educativas.

- La constatación de la irrupción acelerada de los medios de comunicación en la vida social y de su gran poder de conformación de hábitos, actitudes y formas de pensar, lo que los convierte en canales facilitadores de aprendizajes, positivos o negativos, tanto dentro como fuera de la escuela.

- La necesidad de adaptar a los trabajadores a las nuevas necesidades del sistema productivo - los reciclajes, la actualización, la reconversión-que ha propiciado el desarrollo del sector no formal en el mundo del trabajo.

- Los cambios sufridos en el interior de la estructura familiar, que se manifiestan por vía de una delegación de ciertos aspectos del cuidado de los niños en otras instituciones de custodia, educación y recreo infantil -guarderías, ludotecas, colonias de verano, asociaciones juveniles, etc.

- La aparición de un mayor tiempo de libre disponibilidad por reducciones en la jornada laboral, jubilaciones anticipadas, dificultades de acceso al primer empleo e incluso las necesidades que provoca la aparición de una industria del ocio de atraer mayor demanda para incrementar la rentabilidad, son otro conjunto de factores que 
operan en favor de la pujanza de los canales educativos no formales.

- Finalmente se une a todos estos factores mencionados la crisis económica de la institución escolar, cuya contestación obliga a pensar en otras posibles vías de solución.

Un sector importante del proceso global de educación cual es el de la llamada educación no formal, está hoy, pues, presente y a disposición de la demanda social en materia recreativa, instructiva y formativa. Su diversidad es grande en lo concerniente a los destinatarios, objetivos y funciones a los que sirve; el tipo de financiación que los sostiene; las instituciones y organismos que los promueven, etc. Tan heterogéneo panorama revela la complejidad que encierra hacer un estudio como el que tan acertadamente se afronta en esta obra, en la que el autor hace un esfuerzo, en primer lugar, por delimitar el concepto de eso que venimos llamando «educación no formal» para lo cual era casi ineludible confrontarlo con los de educación formal e informal ya que juntos nos permiten diferenciar el conjunto de dispositivos - a veces complementarios, a veces alternativos- a través de cuyo concurso se promueven los aprendizajes de conocimientos, hábitos y actitudes en los ciudadanos. En segundo lugar, a lo largo de varios capítulos, y de forma muy condensada, debido a la ambiciosa amplitud del objeto de estudio de este trabajo, se describe en su globalidad el sector educativo no formal, a través de una enumeración de las funciones, instituciones, medios y métodos de la educación no formal, incluida una clasificación taxonómica que permite ordenarlo desde diversos criterios (destinatarios, personal educador, contexto social, aspectos metodológicos, fuentes de financiación, etc.). Finalmente tomando el concepto de educación permanente como el principio armonizador de la or- ganización global del sistema educativo, y como una aspiración deseable para el futuro, se aborda la interrelación que puede haber entre los sectores formal y no formal; para concluir en la necesidad de que a través de una planificación integradora de ambas instancias se clarifiquen las iniciativas actualmente en marcha, se formulen prioridades en las que deba intervenir cada sector, se evalúen los programas, se coordinen a las entidades y se diseñen programas específicos de educación no formal que tengan en cuenta el medio concreto en que van a realizarse.

Aunque se trata de un trabajo esencialmente descriptivo, el doctor Trilla nos pone también en guardia respecto a la ingenua pretensión de considerar que estamos ante una panacea que va a resolver, en el futuro, los problemas educativos actualmente planteados, tanto en países desarrollados como subdesarrollados. "La educación no formal puede ser tan clasista, alienante, burocrática, ineficaz, onerosa, obsoleta, estática, manipuladora, estereotipada, uniformadora, etc., como lo pueda ser la formal.» Sobre la topografía, tan exhaustivamente hecha, de medios no formales y teniendo como horizonte el derecho a una educación permanente, podemos empezar a preguntarnos a cerca de las virtudes que promete y los vicios que apunta esta nueva disponibilidad de medios con los que aprender.

En resumen, estamos ante un libro que hay que agradecer porque nos va a ayudar a ampliar el horizonte de nuestra reflexión educativa, nos invita a tomar conciencia de la importancia de la parcela no formal de la educación y nos obliga a pensar en la educación como fenómeno humano dentro y fuera de la escuela. 
Capafons, A; Castillejo, J. L.; Gómez Ocaña, C.; Aznar, P., y PéREZ AlONSO-GeTA, P. M. (1985): Autocontrol y Educación. Colección Papers d'Educació, Nau-Libres, Valencia.

A pesar de la corriente de opinión de carácter positivo sobre los estudios de orden interdisciplinar, siguen siendo bastante infrecuentes los trabajos de esta índole en la bibliografía pedagógica. No es el caso, precisamente, de la obra que comentaremos a continuación y que representa un encomiable esfuerzo de integración por parte de algunos autores ubicados en diversos campos de la Teoría de la Educación y de la Psicología, aun cuando sea un departamento universitario de C. C. de la Educación el catalizador de estas inquietudes. Debe aclararse, no obstante, que la finalidad de la obra es explícita $e$ intencionalmente de matiz pedagógico, pues trata -en último término- de ofrecer una tecnología que norme los procesos de aprendizaje del autocontrol. Tal objetivo se resuelve en la elaboración de un estudio monográfico en el que se suman y superponen aproximaciones desde el campo de los estudios de la evolución (en sus vectores filogenético y ontogenético), la antropología, las teorías psicológicas y la educación, enlazadas en un discurso ininterrumpido de fácil lectura y asimilación. Partiendo de la conceptualización de los procesos de autocontrol como objetivo de los programas de intervención educativos y como medio, al mismo tiempo, para conseguir la autonomía personal (uno de los fines últimos de todo proceso educacional) presenta una revisión de las teorías contemporáneas más relevantes sobre la función de autocontrol (Thorensen y Mahoney, Kanfer y Hagerman,
Bandura), a la que sigue un elaborado estudio sobre la autorregulación, autoacomodación y autocontrol en el que destacan los datos aportados por la neurofisiología y la etología en la fundamentación de estos procesos, así como la caracterización del autocontrol como conducta específicamente humana, íntimamente ligada a la función crítica del pensamiento. La perspectiva psicológica sobre el autocontrol, en la que se enumeran y elucidan un buen número de términos relacionados con este proceso, procedentes de diversos enfoques teóricos, junto con un listado de métodos de autodirección completan la obra.

En resumen, una obra completa y equilibrada en la que el lector encontrará una suficiente información para analizar un tema (la programación de actuaciones encaminadas a conseguir sujetos autónomos, conscientes y responsables de sus actos, más independientes del medio en sus decisiones y, por tanto, más libres, en definitiva más humanos) abordado, hasta la fecha, desde posiciones teóricas desprovistas de avales científico-experimentales, cuando no meras declaraciones de intención educadora difícilmente constatables en una práctica en la que padres, maestros, educadores, en general, debían entendérselas amparados exclusivamente en su buen juicio.

$$
\text { VEGA, Felipe }
$$

\section{AA.VV. (1985): Textos de Pedagogia. Conceptos y tendencias en Ciencias de la Educación. Promociones y Publi- caciones Universitarias, Barcelona.}

Elaborar una antología de textos entraña dificultades de diverso orden $y$, por muy bien hechas que estén, se muestran inevitablemente frágiles ante las críticas porque no pueden recogerlo todo 
sin contrariar su significado. Una antología es siempre una selección hecha desde unos criterios, no siempre compartibles, lo cual complica la tarea de satisfacer a todo el que quiera hacer uso de ella. En este caso, sin embargo, estamos seguros de que el libro va a ser acogido con gran interés, por ser un instrumento didáctico de gran ayuda, tanto para los estudiantes que comienzan los primeros cursos de Magisterio y Pedagogía, como para los profesores y estudiosos de temas educativos.

Como se nos advierte, ésta quiere ser «una obra general, introductoria a las Ciencias de la Educación, que recurre directamente a textos y autores que han ido configurando tales disciplinas». El libro recoge, en efecto, textos de autores, la mayoría ya clásicos o expertos muy relevantes en las disciplinas pedagógicas, algunos españoles y otros extranjeros, a los que se han añadido varios elaborados por los propios compiladores. Sería excesivo nombrarlos aquí a todos, pero sirvan como botón de muestra los siguientes: Mantovani, Dewey, Herbart, Durkheim, Althusser, Piaget, Guilford, Rousseau, Marín, García Hoz, Sanvisens, Moore, García Garrido, Escolano, etc. Sin duda, muchos otros merecerían haber sido incluidos, pero como hemos dicho esa es una de las limitaciones impuestas por toda selección, en este caso atemperada por la calidad de los que están.

La temática abordada representa una excelente introducción en el conocimiento científico de la educación en sus aspectos conceptuales, epistemológicos, filosóficos, metodológicos, psicológicos, históricos, tecnológicos, etc. Recoge modelos que van desde la pedagogía tradicional a las tendencias educativas contemporáneas. Se adentra en temas de gran actualidad como son la educación permanente, la educación ambiental, la animación sociocultural y otros. Finalmente están tratados los fundamentos de las principales Ciencias de la Educación.
Otro punto positivo de estos Textos de Pedagogía es la forma en que se nos ofrecen. Cada uno de ellos viene acompañado de una presentación con la mínima información necesaria sobre el autor, su obra y el libro en concreto de donde ha sido extraído el párrafo escogido. Ello permite contextualizar mejor el fragmento de información seleccionado sin que con ello se agote, lo que podría haber sido contraproducente, la posibilidad de una reelaboración posteriór por parte de los alumnos. A la presentación le siguen unas cuestiones, preguntas, ejercicios y sugerencias, que son actividades propuestas para realizar a partir de los textos. Su inclusión no era, desde luego, necesaria, puesto que la utilización de los textos como herramienta metodológica es perfectamente conciliable con estilos docentes muy variados, pero a nadie obligan y sirven para dar ideas. Por último, tras las cuestiones, se ofrece una bibliografía cuya lectura permite seguir profundizando ya sea en el tema, ya sea en el autor.

Quienes se acerquen a esta obra esperando encontrar puntos de referencia que les orienten en los caminos del estudio teórico del hecho educativo, no se sentirán defraudados. Advertirán que la propia lectura de discursos diferentes sobre cuestiones tan variadas les requerirá el esfuerzo de buscar aclaración a las dudas que les surjan, de adivinar los problemas que se derivan de los temas planteados, de valorar los enfoques y las razones que los soportan. Descubrirán, en definitiva, que están ante un instrumento de trabajo que les ayudará a acercarse a la comprensión del hecho educativo en sus dimensiones descriptiva, teórico-práctica y normativa a través de fragmentos claves del pensamiento de expertos, muy relevantes la mayoría, en sus respectivas áreas de conocimiento, dentro de una gama plural de posicionamientos científicos e ideológicos.

VERA VILA, Julio 
Trilla, J. (1985): Ensayos sobre la escuela. El espacio social y material de la escuela. Laerte, Barcelona.

Esta obra es lo que promete en su título, es decir, un análisis riguroso y sugerente, pero sin ánimo de exhaustividad, en torno a la escuela, o más concretamente como indica el subtítulo, en torno al espacio social y material de la escuela. Su núcleo temático cuantitativamente más importante había sido ya publicado en los años 79 y 80 en la revista, hoy desaparecida, El Viejo Topo y se mantiene, según el propio autor, sin modificaciones sustanciales. Este hecho no es, sin embargo, impedimento que reste unidad al texto, sino que, muy al contrario, es prueba de la sistematicidad y coherencia discursiva del autor.

El referente próximo de las tesis mantenidas por el profesor Trilla es la prolífica crítica pedagógica que, desde diversas fuentes y con objetivos heterogéneos, ha sido vertida contra la escuela desde finales de la década de los sesenta, poniendo en entredicho el modelo, los procedimientos, las funciones, la significación u otros aspectos de la que sigue siendo la más representativa de las instituciones educativas de la sociedad actual. La crítica de un espacio segregado de la realidad social e institucionalizado para cumplir unas finalidades educativas, de entre las que destacan la de transmitir los conocimientos sancionados socialmente como deseables y la de desarrollar unas aptitudes, fundamentalmente intelectuales, revela, directa $\mathrm{e}$ indirectamente, la existencia de otros espacios sociales potencialmente educativos donde se encuentra la realidad aludida por los saberes transmitidos. Por ello, cabe hallar un referente remoto de esta obra en el estudio de los espacios educativos no destinados en exclusividad a la tarea formativa, enfoque que traslada el eje del discurso pedagógico desde el ámbito más o menos cerrado, según el modelo pedagógico, de los mu- ros de la escuela al otro mucho más amplio de las relaciones de la especie humana con su entorno cultural, históricamente constituido.

El primer capítulo, que es el único realmente nuevo, es una caracterización de la escuela, un intento de descubrir los rasgos que les son peculiares, por encima de sus diferencias, a los diversos tipos de escuelas. Estos rasgos son:

- El ser una realidad colectiva, un lugar donde se llevan a cabo, en común, diversas actividades relacionadas con la enseñanza y el aprendizaje.

- El ser un espacio específico, un lugar delimitado, más cerrado o más abierto a lo que ocurre más allá de sus límites.

- La temporalización más o menos rígida de las actividades escolares.

- La presencia de los roles de docente y discente.

- La selección, previa a los actos de enseñanza, de los contenidos educativos.

- Y la descontextualización de esos contenidos del lugar original en el que son o bien gestados, o bien aplicados.

El conjunto de estos rasgos caracteriza a la institución escolar, si bien cada modelo los asumirá con métodos, técnicas y dispositivos diferentes según su enfoque pedagógico.

El segundo capítulo es una reflexión entre dos vocaciones, históricamente contradictorias, de la escuela en su forma de comunicación con el espacio social exterior: la una le aboca al aislamiento, en un intento de huida desde lo cotidiano hacia un modelo idealizado de existencia situado en el pasado o en el futuro; la otra le impulsa a debilitar sus fronteras para inmiscuirse, con diferentes grados de convicción, en al circunstancia exterior que le da sentido, llegando incluso a poner en peligro la legitimidad de su propia justificación. 
Se destacan de este análisis, al que nos convoca el autor, dos hechos de gran relevancia para el estudio teórico de la educación actual, uno es el del acelerado debilitamiento de la hegemonía del espacio escolar en beneficio de otros espacios que la tecnología hace posible utilizar pedagógicamente sin que ello suponga, necesariamente, cambio sustancial alguno del paradigma escolar tecnocrático; el otro es la importancia del estudio de la educación informal, de las relaciones y situaciones no específicamente educativas, para comprender y orientar el proceso educativo global, incluida la institución escolar, en toda su dimensión antropológica.

En el tercer capítulo la escuela es vista como instrumento de control social, tanto en sus mecanismos sancionadores de regulación interior, de los que es'arquetipo el tan denostado "examen", como en sus mecanismos externos legales, políticos y sociales. Con todo, no se rechaza la posibilidad de una práctica pedagógica crítica, no exenta de dificultades.

Finalmente se analizan, en el último capítulo, algunas de las repercusiones de las tres propuestas básicas del socialismo clásico (la reivindicación del trabajo infantil, la compatibilidad del trabajo productivo y la enseñanza y la formación polivalente) sobre la escuela, donde, una vez más, la pervivencia de la misma es sometida a prueba, esta vez ante la posibilidad, utópica por el momento, de una sociedad socialista donde habría que repensarla no ya tanto en función de una pedagogía del trabajo cuanto de una pedagogía del tiempo libre.

Completa esta obra una colección de ilustraciones comentadas que ejemplifican muy bien las ideas desarrolladas a lo largo de estos cuatro ensayos, a la vez que quieren ser una invitación a la tarea de emprender una historia de los lugares de la enseñanza. No son una colección de imágenes dispuestas de cualquier modo, sino que, consistentes por sí mismas y dispuestas con un cierto orden cronológico, son comentadas desde el punto de vista de la relación entre espacio escolar y modelo pedagógico. Sólo a modo de ejemplo de lo que el lector puede encontrar, destacaré los siguientes títulos: el orden en el espacio peripatético, el modelo preceptoral, las escuelas de enseñanza mutua, el aula tradcional modélica, el paradigma del activismo, etc.

VERA VILA, Julio

RODRÍGUEZ BORNAETXEA, ADOLFO (1985): Hezkuntzaren teoria, textu eta textuinguruak. Iruñea-Pamplona, Udako Euskal Unibersitatea.

Recientemente celebrábamos en el País Vasco uno de los mayores logros significativos en la producción bibliográfica escrita en euskara, ya que en pocos años se ha conseguido publicar un volumen de obras superior al total de toda la literatura vasca desde sus inicios hasta 1983.

Son muchos los factores que explicarían este fenómeno de tan importante repercusión para la cultura y la educación de un pueblo. Complejidad de un contexto social donde iniciativas públicas y privadas están desarrollando una labor meritoria, si bien para muchos puede parecer de escasa incidencia para la deseada normalización del euskara. Lo cierto es que el trabajo constante e institucional posibilita al lector euskaldun el contacto con obras escritas en su propia lengua.

En este sentido, el libro que comentamos, publicado por U.E.U. (Universidad Vasca de Verano), institución que a nivel universitario tiene entre sus objetivos lograr una universidad en euskara y que lleva tras de sí una ya larga ex- 
periencia de actividades universitarias, es una prueba de esa política de desarrollo cultural, lingüístico y educativo en el terreno del euskara.

Los comentarios que anteceden se hacen casi necesarios para referirnos al libro de Rodríguez Bornaetxea, profesor de Teoría de la Educación de la Facultad de Filosofía y Ciencias de la Educación de la Universidad del País VascoEuskal Herriko Unibertsitatea, Facultad que, dicho sea entre paréntesis, desarrolla una planificación del euskara que alcanza un alto nivel, tanto en el profesorado como en el alumnado, siendo así que son muchas las asignaturas que se imparten en este idioma, además de la existencia de una especialidad única en las universidades del Estado, como es la Pedagogía del Lenguaje que puede cursarse íntegramente en euskara.

El texto que comentamos debe ser entendido en esta doble vertiente: primero como una aportación importante a la escasa producción escrita sobre pedagogía en euskara y segundo como un material de uso didáctico para el alumnado euskaldun (vascoparlante) de una Facultad de Pedagogía. Como el mismo autor señala en la introducción a su libro, se trata de un "lan-tresna" (instrumento de trabajo) para el desarrollo temático de la teoría de la educación.

La obra está estructurada alrededor de una serie de temas a partir de los cuales es posible reflexionar sobre teoría de la educación. Temas que abarcan desde la problemática que plantea el método científico, a cuestiones relacionadas con epistemología de la ciencia, así como materias más específicas en el horizonte educativo como puedan ser la práctica escolar o los fines de la educación. Dichos temas van introducidos por una síntesis resaltando los aspectos más sobresalientes del mismo, o el estado de la cuestión; así mismo se acompañan los temas con una serie muy bien escogida de textos que ayudan a su mejor comprensión. No se trata, pues, de un tra- tado sistemático de teoría de la educación, no es este el objetivo que el autor se plantea, sino de una invitación al estudiante de pedagogía para que observe los límites y posibilidades que la educación ofrece como objeto teórico. Las cuestiones que plantea desde esta perspectiva son sugerentes, si bien hubiésemos deseado una profundización en los temas abordados. Se trata de una visión caleidoscópica que pone en juego tantos elementos que configuran el discurso pedagógico; un texto abierto que se convierte en una herramienta de trabajo necesaria para la reflexión teórica.

Como complemento y para ubicar a los autores de los cuales se trata en el texto se acompaña unas pequeñas notas biográficas. Habrá que decirse, de paso, que muchos de los autores recogidos en la obra ven por primera vez su traducción al euskara, contribuyendo accesoriamente a una selección de textos pedagógicos, que, sin duda, han de facilitar el camino a una deseada biblioteca de obras pedagógicas escritas en euskara.

\author{
DAVILA BALSERA, Pauli \\ (Universidad del País Vasco- \\ Euskal Herriko Unibertsitatea)
}

Castillejo, J. L.; Claudio, A.; EscáMEZ, J.; GARCÍA, R.; GARRIDO, V., y MARTÍNEZ, B. (1986): Conceptos y propuestas (III). Nau Llibres, Valencia.

El presente volumen es el tercero de la colección «Conceptos y Propuestas» sobre cuestiones de teoría de la Educación que publica el departamento de «Fundamentos de la Educación» de la Universidad de Valencia. José Luis Castillejo aborda el tema de la incidencia de la educación en la construcción humana; después de revisar el problema de la herencia-medio abandona, por insufi- 
ciente y esclerotizado, el modo tradicional de plantearlo, sobre qué elemento del binomio tenga más peso en la conformación de la personalidad, señalando que el «hombre no es ni su herencia ni su medio sino el complejo producto de ambas variables, de modo que sólo van a operativizarse algunos descriptores genéticos, mientras que otros permanecerán siempre cerrados, según los vectores de estimulaciones externas que intervengan». Desde esta perspectiva la educación en una de las claves para operativizar y dinamizar determinados descriptores genéticos cuyo desarrollo es deseable e inhibir aquellos que sean considerados perjudiciales. La ciencia pedagógica como intervención técnica sobre las condiciones que permitan uno $u$ otro proceso se construye en una actividad científicamente rigurosa y humanamente relevante. Juan Escámez se plantea la presencia/ausencia de los valores en la pedagogía de la intervención. El problema se formula así: ¿Al tecnólogo de la educación le compete juzgar si un determinado patrón (objetivo o meta educativa) es valioso? Después de analizar lo que es la tecnología educativa, el ámbito de su aplicación y la función social que como profesional, desempeña el tecnólogo de la educación, concluye que los valores no pueden estar ausentes en la intervención educativa; además plantea las interacciones y diferencias entre la pedagogía y la axiología, resaltando su profunda interacción. Rafaela García realiza un estudio sobre los elementos básicos de la acción educativa, delimitando aquellos que la diferencian para concluir que presenta peculiaridades en relación con otros tipos de acción; para ello la analiza en los diferentes ámbitos de las ciencias humanas. Amalia Claudio centra su trabajo en la delimitación de «los conceptos de ambiente y educación» por contraposición a otros conceptos análogos. A partir de ahí estudia los distintos ambientes, su operativización y organización para influir en el proceso educativo. Bernardo Martínez Mut nos expone los principios que animan la Tería $Z$, que se ha mostrado eficaz tanto económica como humanamente en la industria, y su aplicación a las instituciones educativas. Después de señalar las razones que justifican dicha aplicación, extrae las características fundamentales que una escuela por ella animada ofrecería, así como las estrategias de intervención que habrían de seguirse. Por último, Vicente Garrido hace un análisis crítico a los modelos de reintegración vigentes en las prisiones españolas, mostrando sus lagunas e insuficiencias para desde ahí propugnar un nuevo modelo de reintegración donde los procedimientos derivados de la psicología del aprendizaje y de la educación son centrales.

GOMEZ-OCAÑA, Concha

Puig, Josep M.', y Trilla, Jaume (1985): Pedagogia de l'oci. CEAC, Barcelona (texto catalán).

Hace escasamente unos años, el lector que se acercara a las bibliotecas o librerías en busca de textos dedicados al tema del ocio, el tiempo libre o la animación sociocultural, raramente podría conseguir mucho más que un pequeño número de obras, generalmente de carácter muy específico: descripción de algunas experiencias realizadas por instituciones públicas o privadas o con títulos relativos a tratados o reglamentos deportivos, de manualidades o dinámica de grupos. Rara vez se encontraban correctamente clasificados, su selección era mediocre y los manuales $u$ obras generales brillaban por su ausencia. Afortunádamente, el interés despertado por estos temas en las instituciones estatales, los estudiosos de diversas ciencias y la población, en general, ha permitido y fomentado la publicación, recientemente, de libros con 
mayor amplitud y profundidad temática.

Josep M.' Puig y Jaume Trilla presentan en «Pedagogia de l'oci» (vencedor del «VII Premi d'Educació Josep Pallach») una visión totalizadora de este nuevo ámbito de la Pedagogía. Un estudio sistemático de un campo donde muchos activistas - pedagogos, inclusose mueven sin rumbos claros, a falta de un modelo interpretativo, como el que este libro presenta.

Partiendo de la delimitación conceptual del ocio (distinguiéndolo, relacionándolo con otros términos cercanos, tiempo libre, por ejemplo), estudian en los primeros capítulos el desarrollo histórico del concepto, las teorías contemporáneas, así como los precedentes y razones objetivas que hicieron germinar esta nueva parcela de las Ciencias de la
Educación. Reflexiones en torno a aspectos teóricos de la p. del ocio, a las condiciones de las intervenciones en muy diversos ámbitos (específicos y noespecíficos de esta disciplina, que cubren en su conjunto la práctica totalidad del sistema educativo informal), forman el grueso de esta interesante obra.

Una visión completa, un modelo teórico-práctico que ayudara no poco a profesores, educadores, animadores culturales... una obra que, como menciona el doctor Sanvisens en la presentación de la misma, puede calificarse de Pedagogía Social, en su concepción más amplia que agrupa la educación social y la labor educativa de la sociedad.

VEGA, Felipe 